\title{
Relationship between Features of Augmented Reality and User Memorization
}

\author{
Yuichiro Fujimoto Goshiro Yamamoto Takafumi Taketomi Jun Miyazaki Hirokazu Kato * \\ Nara Institute of Science and Technology
}

\begin{abstract}
The objective of this study is to investigate the relationship between the features of augmented reality (AR) and human memorization ability. The basis of this relation is derived from the following features. The AR feature is that AR can provide information associated with specific locations in the real world. The feature of human memory is that humans can easily memorize information if the information is visually associated with specific locations. To investigate this relation, we conduct a pilot user study in which blocks are picked from some drawers. As a result, significant differences are found between a situation in which visual information is displayed at the location of each drawer in the real world and that in which textual information is displayed at an unrelated location.
\end{abstract}

Index Terms: H.1.2 [Models and Principles]: User/Machine Systems-Human factors; H.5.1 [Information Interfaces and Presentation]: User Interfaces-Artificial, augmented and virtual reality

\section{INTROdUCTION AND RELATED WORK}

Supporting human tasks in the real world is one of the most important objectives of augmented reality (AR). The usefulness of AR systems which support users' task has been widely researched. In this paper, we evaluate the relationship between features of AR and the user's memorization. AR is the technology that overlays information about a specific location on the user's real-world view. Users may perceive not only the information itself but also its location. Thus, information displayed by AR can be regarded as naturally containing real-world locations. It is also known that humans can easily memorize and retain information if this information is visually associated with real-world locations [1][2]. Therefore, we hypothesized that annotations displayed using AR may augment the user's memory skill if the annotations are associated with the realworld location of the target object instead of an unrelated location. If the hypothesis can be proven, we could argue that task-support systems using AR are effective for not only providing information related to tasks but also facilitating memorization of tasks.

Tang et al. stated that the features of AR have positive effects on block assembly tasks in terms of supporting human spatial cognitive ability [3]. Schwerdtfeger et al. compared a situation with 'AR instruction in situ', one with 'text-based list' and so on for a users' picking task in terms of working time and the number of mistakes [4]. Ikeuchi et al. showed that overlaying symbols on pictures of specific locations in the real world with a wearable system enabled users to memorize these pictures and retain them longer than usual [2]. However, it remains to be verified how AR display methods affect users' memorization when the users are instructed to memorize and recall information displayed by AR.

As a first step, we conducted a user study in [5], and we verified that displaying annotations using AR can enhance a user's memory

*e-mail: \{yuichiro-f, goshiro, takafumi-t, miyazaki, kato\} @is.naist.jp

IEEE International Symposium on Mixed and Augmented Reality 2012 Science and Technology Proceedings

5 - 8 November 2012, Atlanta, Georgia

978-1-4673-4662-7/12/\$31.00 @2012 IEEE skill in a task in which users are instructed to memorize individual symbols, if the annotations are displayed near the real-world location of the target object instead of an unrelated location. In this paper, we conducted another user study in which users were instructed to memorize continuous task processes of more realistic tasks, by picking blocks from some drawers in order to evaluate the relation between the features of AR and user memorization.

\section{HUMAN MEMORY SYSTEM}

The ease of memorizing information associated with a specific location is a well-known feature of short-term memory (STM) [1][2]. One of reasons for this ease is the presence of spatial memory. Humans have hundreds of thousands of pyramidal cells used to represent spatial locations called spatial memory. Spatial memory works at the same time as other memories and reinforces the user's memorization. Thus, information located at a specific location is considered to be memorized easily. The usefulness of this memory can be proven by the fact that "the method of loci" is widely used as a memorization technique [6]. If this feature is applied to AR, we can assume that a user may easily recall displayed objects (information) that are visually associated with specific locations, as compared to those that are not.

\section{USER STUdY}

We evaluated the relation between the features of AR and memorization when users were instructed to memorize a continuous task process. Users were instructed to memorize the task of picking some blocks from each of nine drawers in the correct order.
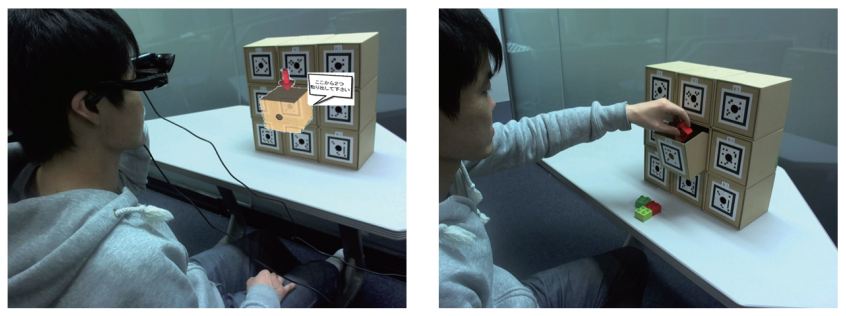

Figure 1: Overview of the experimental steps: (Left) The step in which a user memorizes each task process. (Right) The step in which a user recalls each process and picks some blocks from each drawer in the correct order.

\subsection{Experimental System}

The user device consists of an HMD (video-see-through type) equipped with a single web-camera. The HMD provides $640 \times 480$ images at $30 \mathrm{fps}$. Users wear the HMD and all the information is displayed using AR. Each of the nine drawers contains 10 blocks, and users are required to pick some of them from the drawers in the correct order. The drawers are labeled with AR markers and alphanumerical labels that distinguish each location, such as 'L1' (first one in the left column) or 'M2' (second one in the middle column). Twelve task instructions are displayed for users in turn as one task annotation, which indicates "how many blocks" are to be taken 
and "from which drawers" they are to be picked. After displaying for 10 seconds, each step is automatically switched to the next step. Users are instructed to memorize "how many blocks" are taken and "from which drawers" they are picked while each step annotation is being displayed. After the memorization session, they were instructed to remove the HMD and wait for 30 seconds to facilitate fading of the short-term memory of the session. After this clearing session, the users were instructed to recall the task process and to pick blocks from each drawer in the correct order. These procedures were repeated for the following two different display methods.

Figure 1 shows the experimental steps. The figure on the left shows the step in which a user memorizes each task process. The figure on the right shows the step in which a user recalls each process and picks some blocks from each drawer in the correct order.

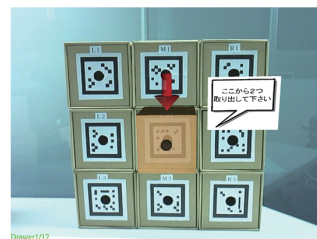

(a) Type 1

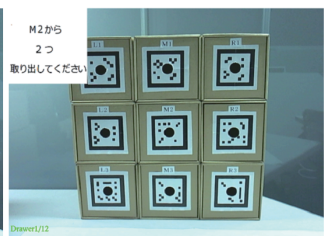

(b) Type 2
Figure 2: Display methods in the user study

\section{Information Display Method}

The information was displayed in the system using the following two display methods.

Type 1: The information is displayed near the location of each drawer (with visual and textual instruction).

Figure 2(a) shows this display method. In the method, a computer generated (CG) drawer, arrow and textual annotation indicating "how many blocks" to take are displayed near the location of each drawer. CG drawers and arrows indicate "from which drawers" visually. This display method exploits the AR feature, which can visually associate information with a specific location in the real world.

Type 2: The information is displayed at a fixed location (with only textual instruction).

Figure 2(b) shows this display method. In the method, only a textual annotation is displayed at a fixed location without regard for the location of the drawer. This textual annotation indicates both "how many blocks" and "from which drawers". The location of each drawer is displayed as the labeling number marked on the drawers, e.g., 'L1' or 'M2'. This display method simulates the situation in which target objects and information are memorized separately without using AR, such as when a user memorizes task processes by referring to a paper list of the task processes.

\subsection{Experimental Design}

Sixteen males and 2 females, 23 to 27 years old (average 24.4), participated in the study. We used a within-subjects design for arranging the two display methods. We predicted that users will memorize both "how many blocks" and "from which drawers" more accurately with display method Type 1 than with Type 2 .

\section{Experimental Results and Consideration}

Figure 3 shows the accuracy rate of the test answer for each display method. Figure 3(a) shows the accuracy of "from which drawers" and figure 3(b) shows the accuracy of "how many blocks". With a paired t-test, it was found that the users memorized them more accurately with Type 1 than with Type 2 ("from which drawers": $\mathrm{t}(17)=2.29, \mathrm{p}<.05$, "how many blocks": $\mathrm{t}(17)=2.82, \mathrm{p}<.05)$.

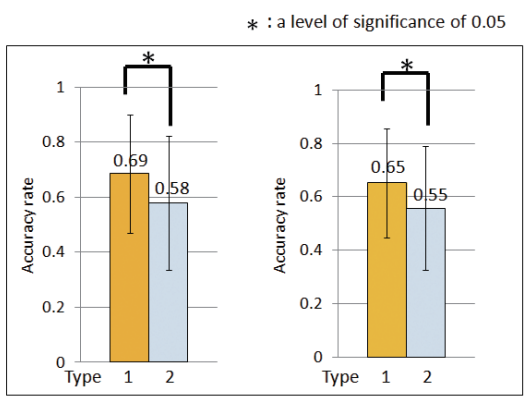

(a)

(b)

Figure 3: Accuracy rate comparison of the two display methods, (a, Left): the accuracy of "from which drawers" (b, Right): the accuracy of "how many blocks".

In general, visual instructions affect positively memorization for allowing users to intuitively understand them. For "from which drawers", visual instructions are used with Type 1 and textual instructions are used with Type 2 . The significant difference is considered to be reasonable. On the other hand, for instructing "how many blocks", textual instructions are used in both Type 1 and 2. It is known that when humans memorize an object and its location, his or her memorization process is disturbed, causing his or her attention to visually shift to unrelated locations [7]. Type 1 might have less disturbance for the user's memorization than Type 2 for difference of displaying locations. To support the effectiveness of display location, however, we have to conduct a follow-up user study comparing it with another display method, such as one using actual paper with visual instructions. At least we can conclude that using AR and displaying visual instructions associated with each location is more effective for memorization than displaying textual instructions at a fixed location.

\section{Conclusion ANd Future Work}

Results showed that displaying visual instructions at the locations to be memorized is useful for memorizing them visually, as in the case of the user study in which individual semantic memory was used as the memorization target [5].

In the future, we plan to conduct a follow-up user study to separately evaluate the effect of instructions in place or not, and the effect of visual or textual instructions.

\section{REFERENCES}

[1] K. A. Ericsson. "Memory skill", Canadian Journal of Psychology, 1985.

[2] Y. Ikei and H. Ota. "SROM: Spatial Electronic Mnemonics : Basic concepts and the characteristics of registration process", Journal of The Virtual Reality Society of Japan 14(2), pp.241-249, 2009 (in Japanese).

[3] A. Tang, C. Owen, F. Biocca, and W. Mou. "Comparative Effectiveness of Augmented Reality in Object Assembly", In Proc. the SIGCHI conference on Human factors in computing systems, pp.73-80, 2003.

[4] B. Schwerdtfeger, T. Frimor, D. Pustka, and G. Klinker. "Mobile information presentation schemes for logistics applications", In Proc. 16th International Conference on Artificial Reality and Telexistence (ICAT 2006), November 2006.

[5] Y. Fujimoto, G. Yamamoto, J. Miyazaki, and H. Kato. "Relation between Location of Information Displayed by Augmented Reality and User's Memorization", 3rd Augmented Human International Conference, pp.93-100, 2012.

[6] D. O'Brien. "Learn to Remember", Duncan Baird Publisher, 2000.

[7] H. D. Zimmer, H. R. Speiser, and B. Seidler. "Spatio-temporal working-memory and short-term object-location tasks use different memory mechanisms", Acta Psychologica, Vol. 114(1), pp.41-65, 2003. 